\title{
Wnt7A Identifies Embryonic $\gamma$-Motor Neurons and Reveals Early Postnatal Dependence of $\gamma$-Motor Neurons on a Muscle Spindle-Derived Signal
}

\author{
Soha Ashrafi, ${ }^{1,2}$ Melanie Lalancette-Hébert, ${ }^{3}$ Andreas Friese, ${ }^{4,5}$ Markus Sigrist, ${ }^{4,5}$ Silvia Arber, ${ }^{4,5}$ Neil A. Shneider, ${ }^{3}$ \\ and Julia A. Kaltschmidt ${ }^{1,2,6}$ \\ ${ }^{1}$ Neuroscience Program, Weill Cornell Medical College, New York, New York 10065, ²Developmental Biology Program, Sloan-Kettering Institute, New York, \\ New York 10065, ${ }^{3}$ Department of Neurology, Center for Motor Neuron Biology and Disease, Columbia University, New York, New York 10032, ${ }^{4}$ Biozentrum, \\ Department of Cell Biology, University of Basel, CH-4056 Basel, Switzerland, ${ }^{5}$ Friedrich Miescher Institute for Biomedical Research, CH-4058 Basel, \\ Switzerland, and ${ }^{6}$ Cell and Developmental Biology Program, Weill Cornell Medical College, New York, New York 10065
}

Motor pools comprise a heterogeneous population of motor neurons that innervate distinct intramuscular targets. While the organization of motor neurons into motor pools has been well described, the time course and mechanism of motor pool diversification into functionally distinct classes remains unclear. $\gamma$-Motor neurons $(\gamma$-MNs) and $\alpha$-motor neurons $(\alpha$-MNs) differ in size, molecular identity, synaptic input and peripheral target. While $\alpha$-MNs innervate extrafusal skeletal muscle fibers to mediate muscle contraction, $\gamma$-MNs innervate intrafusal fibers of the muscle spindle, and regulate sensitivity of the muscle spindle in response to stretch. In this study, we find that the secreted signaling molecule Wnt7a is selectively expressed in $\gamma$-MNs in the mouse spinal cord by embryonic day 17.5 and continues to molecularly distinguish $\gamma$-from $\alpha$-MNs into the third postnatal week. Our data demonstrate that Wnt7a is the earliest known $\gamma$-MN marker, supporting a model of developmental divergence between $\alpha$ - and $\gamma$-MNs at embryonic stages. Furthermore, using $W n t 7 a$ expression as an early marker of $\gamma$-MN identity, we demonstrate a previously unknown dependence of $\gamma$-MNs on a muscle spindlederived, GDNF-independent signal during the first postnatal week.

\section{Introduction}

The precise temporal and spatial activation of different muscles is critical for the generation of animal behavior. Motor neurons in the spinal cord play a fundamental role in coordinating muscle activity by mediating the transfer of information from sensory neurons, long-range projection neurons, and interneurons to distinct muscle groups in the periphery (Kanning et al., 2010). Motor neurons that innervate a single muscle group are arranged in the spinal cord into units called motor pools (Romanes, 1951).

\section{Received March 8, 2012; accepted May 3, 2012.}

Author contributions: S. Ashrafi, S. Arber, N.A.S., and J.A.K. designed research; S. Ashrafi, M.L.-H., M.S., and N.A.S., performed research; A.F., S. Arber, and N.A.S. contributed unpublished reagents/analytic tools; S. Ashrafi, S. Arber, and J.A.K. analyzed data; S. Ashrafi, S. Arber, N.A.S., and J.A.K. wrote the paper.

A.F., M.S., and S. Arber were supported by a European Research Council Advanced Grant, the Swiss National Science Foundation, the Kanton Basel-Stadt, European Union (EU) Framework Program 7, and the Novartis Research Foundation. M.L-H. and N.A.S. were supported by grants from the National Institutes of Health (R21 NS072573) and the Motor Neuron Center at Columbia University; J.A.K. was funded by Memorial Sloan-Kettering start-up funds, a Whitehall Foundation Research Grant, and a Louis V. Gerstner, Jr. Young Investigators Award. We are grateful to Zaiqi Wu and Frank Costantini for providing Ret mutant mice. We also thank Susan Morton and Thomas Jessell for providing unpublished rabbit anti-ChAT and guinea pig anti-Islet1 antibodies, and Dritan Agalliu and Jan Kitajewski for providing Wnt7a antisense probe. We thank Thomas Jessell and Peter van Roessel for comments on the manuscript.

The authors declare no competing financial interests.

Correspondence should be addressed to Julia A. Kaltschmidt, Developmental Biology Program, Sloan-Kettering Institute and Neuroscience and Cell and Developmental Biology Programs, Weill Cornell Medical College, New York, NY 10065.E-mail: kaltschj@mskcc.org.

A. Friese's present address: Life Technologies, Frankfurter Strasse 129b, 64293 Darmstadt, Germany.

DOI:10.1523/JNEUROSCI.1160-12.2012

Copyright $\odot 2012$ the authors $\quad 0270-6474 / 12 / 328725-07 \$ 15.00 / 0$
Motor neurons within one motor pool can be further subdivided into motor neuron classes that innervate distinct targets within the muscle. $\alpha$-Motor neurons ( $\alpha$-MNs) are the most abundant class of motor neurons within a motor pool. They innervate extrafusal muscle fibers and are responsible for activating skeletal muscle fibers to generate force. $\alpha$-MNs can be further subdivided into fast fatigable, fast fatigue-resistant and slow twitch motor neuron subtypes based on the contractile and metabolic properties of the muscle fibers they innervate (Kanning et al., 2010). $\gamma$-Motor neurons ( $\gamma$-MNs), representing $\sim 30 \%$ of motor neurons within a pool, innervate spindle intrafusal muscle fibers, and function in the regulation and adjustment of proprioceptive input to the CNS (Kuffler et al., 1951; Burke et al., 1977). During postnatal stages, $\gamma$ - and $\alpha$-MNs can be further distinguished based on their size and the amount of sensory input they receive. $\gamma$-MNs have small cell bodies and receive little proprioceptive sensory input, whereas $\alpha$-MNs are larger and most receive direct input from proprioceptive sensory afferents (Kanning et al., 2010).

Recent studies have sought to identify molecular markers differentially expressed between the various subpopulations of neurons within a motor pool. Molecular markers that differentiate between fast and slow $\alpha$-MNs have been described in postnatal development (Chakkalakal et al., 2010; Enjin et al., 2010). However, while the presence of a distinct $\gamma$-MN population during embryonic development has been inferred (Gould et al., 2008; Sabharwal et al., 2011), there are no known markers capable of 
differentiating $\gamma$ - and $\alpha$-MNs before postnatal stages. Given the absence of embryonic markers for $\gamma$-MNs, the developmental time course and mechanism by which these motor neurons obtain their distinct class identities remain unclear. Also, recent studies have shown that the survival of $\gamma$-MNs depends on the expression of GDNF by muscle spindles in the second postnatal week (Shneider et al., 2009). However, due to the inability to distinguish $\gamma$ - from $\alpha$-MNs during embryonic and neonatal development, it has been unclear whether muscle spindle-derived signals might also play an earlier role during $\gamma$-MN development. Here we show that Wnt7a expression marks $\gamma$-MNs neurons at embryonic day 17.5 (E17.5), thereby making it the earliest known $\gamma$-MN marker. Using this marker we demonstrate that $\gamma$-MNs depend on a muscle spindle-derived, GDNF-independent signal during early postnatal development.

\section{Materials and Methods}

Mouse lines. Egr3 $3^{-1-}$ (Tourtellotte and Milbrandt, 1998), Gfr $\alpha 1^{-1-}$ (Enomoto et al., 1998), Gfro1::TLZ (Enomoto et al., 2004), Hb9::GFP (Wichterle et al., 2002), Isl2::DTA (Yang et al., 2001), Pva::Cre (Hippenmeyer et al., 2005), Ret ${ }^{\text {tmlCos }}$ (Schuchardt et al., 1994), Ret ${ }^{\text {CreERT2 }}$ (Luo et al., 2009), and Wnt7a ${ }^{-1-}$ (Parr and McMahon, 1995) were described previously. Both male and female mice were used in this study. $N \geq 3$ mice for all experiments.

Immunohistochemistry. Immunohistochemistry on $12 \mu \mathrm{m}$ thick cryostat sections of spinal cord was performed as previously described (Friese et al., 2009). The following antibodies were used: chick anti- $\beta$ galactosidase (Abcam), goat anti-ChAT (Millipore), rabbit anti-ChAT (generously provided by Susan Morton and Thomas Jessell, unpublished), mouse anti-Err3 (PPMX), rabbit anti-GFP (Invitrogen), sheep anti-GFP (Biogenesis), guinea pig anti-Islet 1 (generously provided by Susan Morton and Thomas Jessell, unpublished), mouse anti-NeuN (Millipore), and rabbit anti-Pea3 (Peljto et al., 2010).

Histochemistry. Slides for immunohistochemistry combined with in situ hybridization for Wnt7a (Agalliu et al., 2009) or ChAT (Arber et al., 1999) were first processed for in situ hybridization as described by Arber et al. (1999), omitting the Proteinase K degradation step. For Wnt7a in situ hybridizations, slides were left for two nights in developing solution and then washed once in PBS before processing for immunohistochemistry.

Area measurements of motor neuron cell bodies. Z-stacks of motor neurons were obtained on a Leica TCS SP5 confocal using $0.5 \mu \mathrm{m}$ optical sections. Briefly, the center of each ChAT-labeled motor neuron was approximated based on the $z$-plane where the nucleus appeared largest. The cross-sectional area of the cell was then measured using Leica LAS software plug-in (Version 2.3.1 build 5194). We restricted our analysis to ChAT-positive $\left(\mathrm{ChAT}^{\mathrm{ON}}\right)$ motor neurons in the lateral motor column (LMC).

\section{Results}

\section{Wnt7a is expressed in $\gamma$-MNs}

Previous studies demonstrated that $\gamma$ - and $\alpha$-MNs acquire different molecular features during early postnatal development (Friese et al., 2009; Shneider et al., 2009). To find markers that label $\gamma$-MNs during embryonic development, we analyzed candidates from an independently performed microarray screen (Friese et al., 2009). We first used in situ hybridization to analyze transcript expression at postnatal stages when the identity of $\gamma$-MNs can most clearly be distinguished. At postnatal day 15 (P15), we found that Wnt7a is expressed by a subset of cells in the ventral horn. To assess whether the Wnt7a-positive $\left(W n t 7 a^{O N}\right)$ cells are motor neurons, we correlated the expression of Wnt7a transcript with that of the motor neuron marker choline acetyltransferase (ChAT). Wnt7a $a^{O N}$ cells were scattered within the area of ventral $\mathrm{ChAT}^{\mathrm{ON}}$ motor neurons at all rostral-caudal levels of the lumbar spinal cord (Fig. $1 A-A^{\prime \prime}$; data not shown). We analyzed whether Wnt7a and ChAT expression overlapped and found that $\sim 84 \%$ of $W n t 7 a^{O N}$ cells colabeled with ChAT ( $n=$ 114 of 136 lumbar LMC neurons; Fig. $\left.1 A^{\prime \prime}, B-B^{\prime \prime}\right)$. Based on these findings we conclude that the majority of $W n t 7 a^{O N}$ cells are motor neurons.

$\gamma$ - and $\alpha$-MNs differ in their relative abundance and cell size (Burke et al., 1977). $\gamma$-MNs are small and comprise $\sim 30 \%$ of all motor neurons, while the remainder are presumed to be mainly large $\alpha$-type MNs (Friese et al., 2009; Shneider et al., 2009). To determine whether $W n t 7 a^{O N}$ cells in the ventral spinal cord are $\gamma$-MNs, we first analyzed the fraction of motor neurons (ChA$\mathrm{T}^{\mathrm{ON}}$ ) that coexpressed $W n t 7 a$. We found that at $\mathrm{P} 15, \sim 22 \%$ of all motor neurons expressed Wnt7a $\left(n=114\right.$ of $526 \mathrm{ChAT}^{\mathrm{ON}}$ motor neurons) and that $W n t 7 a^{O N}$ motor neurons were distinctly smaller than the corresponding $W n t 7 a^{O F F}$ fraction $\left(W n t 7 a^{O N}\right.$ : $395 \pm 194 \mu \mathrm{m}^{2} ; W n t 7 a^{O F F}: 676 \pm 254 \mu \mathrm{m}^{2}$; \pm SD), consistent with their identity as $\gamma$-MNs. To further characterize $W n t 7 a^{O N}$ motor neurons, we used several specific molecular markers known to distinguish $\gamma$ - from $\alpha$-MNs at postnatal stages. The orphan nuclear hormone receptor Err3 is initially expressed broadly in motor neurons and then becomes restricted to $\gamma$-MNs by about P14 (Friese et al., 2009). Another selective marker, the GDNF receptor Gfr $\alpha 1$ becomes gradually restricted to $\gamma$-MNs during the first three postnatal weeks, however, while it labels $89 \%$ of $\gamma$-MNs at P21, it remains weakly expressed in large $\alpha$-MNs (Shneider et al., 2009). We assessed the status of Err3 expression in $W n t 7 a^{O N}$ motor neurons and found that, at P15, $90 \%$ of $W n t 7 a^{O N}$ motor neurons expressed Err3 $(n=103$ of 114 $\mathrm{ChAT}^{\mathrm{ON}} / \mathrm{Wnt}_{7 a^{\mathrm{ON}}}$ motor neurons; Fig. $\left.1 C-C^{\prime \prime}\right)$. Conversely $\sim 75 \%$ of $\mathrm{ChAT}^{\mathrm{ON}} / \mathrm{Err}^{\mathrm{ON}}$ motor neurons expressed $W n t 7 a$ ( $n=103$ of $138 \mathrm{ChAT}^{\mathrm{ON}} / \mathrm{Err}^{\mathrm{ON}}$ motor neurons). Using LacZ immunocytochemistry on a Gfr 1 ::LacZ knock-in line (Enomoto et al., 2004), we also found partial coexpression of $W n t 7 a^{\text {ON }}$ with LacZ at P21 (Fig. $1 D-D^{\prime \prime}$ ). In addition to assessing markers that are restricted to $\gamma$-MNs, we probed those that are excluded from $\gamma$-MNs. $\gamma$-MNs express no or very low levels of the nuclear neuronal protein NeuN (Friese et al., 2009; Shneider et al., 2009), and downregulate the Hb9::GFP transgene, which drives expression of GFP in motor neurons and ventral interneurons (Wichterle et al., 2002; Shneider et al., 2009). Analysis of these negative markers showed that from P5 to P21, Wnt7a ON cells excluded both NeuN (Fig. 1E-E"; and data not shown) and Hb9::GFP (Fig. $1 F-F^{\prime \prime}$; and data not shown). Together, these data demonstrate that $W n t 7 a$ is a novel marker of $\gamma$-MNs in the early postnatal mouse spinal cord.

\section{Wnt7a is the earliest known marker for $\boldsymbol{\gamma}$-MNs}

To determine whether Wnt7a may serve as a marker of embryonic $\gamma$-MNs, we analyzed the pattern of Wnt7a expression in the developing mouse embryo. At E9.5, Wnt7a is expressed in a gradient along the dorsal-ventral axis of the ventricular zone (Agalliu et al., 2009). In E15.5 Hb9::GFP spinal cords, $W n t 7 a^{O N}$ cells were restricted to the ventricular region (Fig. $2 A-A^{\prime \prime}$ ), and $1 \mathrm{~d}$ later, we observed $W n t 7 a^{O N}$ cells in the ventral spinal cord, as well as some remaining cells in the ventricular region (Fig. $2 B, B^{\prime \prime}$ ). Interestingly, at this stage, ventral $W n t 7 a$ expression was limited to cells expressing Hb9::GFP at a relatively low level (Fig. $2 B^{\prime \prime}$ ). Only weak expression of $W n t 7 a$ was observed in the ventricular zone at E17.5, while Wnt7a and Hb9::GFP were expressed in two distinct and intermingled cell populations in the ventral motor nucleus, which we denote as $\mathrm{Hb} 9:: \mathrm{GFP}^{\mathrm{OFF}} / \mathrm{Wnt} 7 a^{\mathrm{ON}}$ and $\mathrm{Hb} 9:: \mathrm{GFP}^{\mathrm{ON}} / \mathrm{Wnt7a^{ \textrm {OFF } }}$ cells (Fig. $2 C, C^{\prime}, E, F$ ). To determine whether Hb9::GFP ${ }^{\mathrm{OFF}} / W n t 7 a^{\mathrm{ON}}$ cells correspond to $\gamma$-MNs at this stage, we took a genetic approach and tested the expression of 

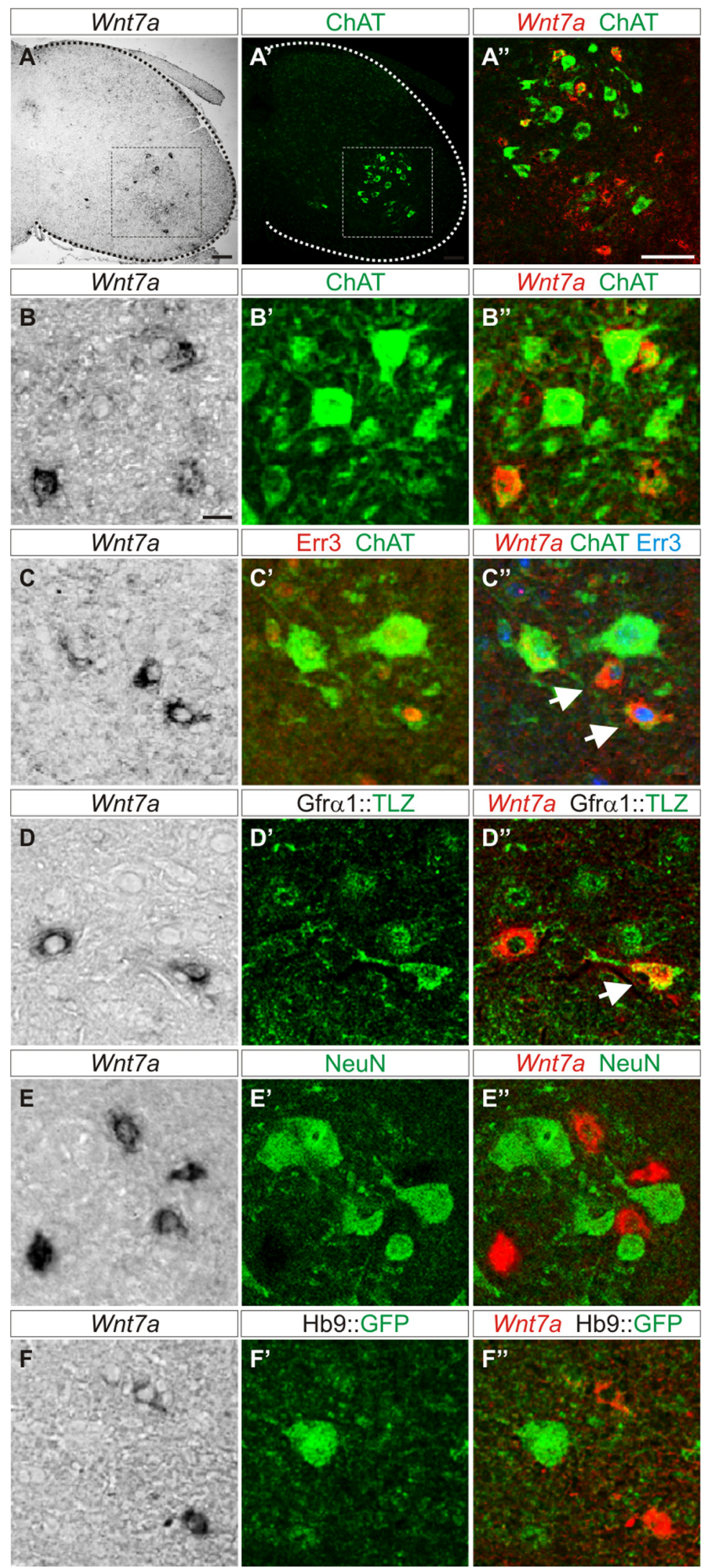

Figure 1. Wnt7A is a $\gamma-\mathrm{MN}$ marker. $A-A^{\prime \prime}, W n t 7 a^{O N}$ cells $(A)$ are located within the $C h A T^{0 N}$ motor nucleus $\left(A^{\prime}\right)$ in the lateral ventral horn of P15 mouse spinal cord. $\boldsymbol{A}^{\prime \prime}$, Magnification of boxed region in $\boldsymbol{A}$ and $\boldsymbol{A}^{\prime}$ showing colabeling of Wnt7a with a subset of ChAT ${ }^{\mathrm{ON}}$ cells.

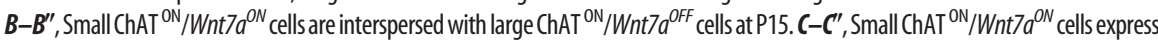
Err3 (arrows) at P15. D- $\boldsymbol{D}^{\prime \prime}$, Small Wnt7 ${ }^{O N}$ cells express LacZ (arrow) in P21 Gfr $\alpha 1:$ :LacZ reporter mice. $\boldsymbol{E}-\boldsymbol{E}^{\prime \prime}$, Wnt7 $a^{\text {ON }}$ cells exclude NeuN atP21.F- $\boldsymbol{F}^{\prime \prime}, W n t 7 a^{\text {ON }}$ cells exclude Hb9::GFP atP21. Split and merged channels shown for $\boldsymbol{B}-\boldsymbol{F}^{\prime}$. Scale bars: $\boldsymbol{A}-\boldsymbol{A}^{\prime \prime}, 100 \mu \mathrm{m} ; \boldsymbol{B}-\boldsymbol{F}^{\prime \prime}, 20 \mu \mathrm{m}$.
Wnt7a in Gfro1 mutant mice (Enomoto et al., 1998) in which $30 \%$ of motor neurons (presumed to be $\gamma$-MNs) are lost by E17.5 (Gould et al., 2008). We intercrossed Gfr $\alpha 1^{-1-}$ mice with the Hb9::GFP transgenic line and showed that at E17.5 $\mathrm{Hb} 9:: \mathrm{GFP}^{\mathrm{OFF}} / W n t 7 a^{\mathrm{ON}}$ cells were absent, while Hb9::GFP ${ }^{\mathrm{ON}} / W n t 7 a^{\mathrm{OFF}}$ cells persisted (Fig. $2 D, D^{\prime}$ ). This finding suggests that $\mathrm{Hb} 9:: \mathrm{GFP}^{\mathrm{OFF}} / \mathrm{Wnt7} a^{\mathrm{ON}}$ status can be used to identify $\gamma$-MNs at E17.5.

In addition to $G$ fr $\alpha 1^{-/-}$mice, we also examined mice deficient in the GDNF receptor, Ret. In E16.5 Ret mutant embryos, almost all muscle spindles are devoid of motor input, suggesting that $\gamma$-MNs are lost at this stage (Gould et al., 2008). We analyzed E17.5 Ret mutant mice (Ret ${ }^{\text {CreERT2/- }}$ ) and found that $90 \%$ of $W n t 7 a^{O N}$ cells were lost (control = 156 Wnt7a $a^{\text {ON }}$ cells; Ret $^{\mathrm{CreERT2/-}}=$ $18 W n t 7 a^{O N}$ cells; Fig. $2 G, H$ ), while putative ChAT ${ }^{O N} \alpha$-MNs persisted (Fig. $2 G^{\prime}, H^{\prime}$ ). Together these data provide further evidence that $W n t 7 a^{O N}$ cells at E17.5 are putative $\gamma$-MNs.

\section{A novel requirement for a muscle spindle-derived signal in early postnatal $\gamma$-MN development}

The expression of Wnt7a in $\gamma$-MNs at late embryonic and early postnatal stages enabled us to explore the role for muscle spindle-derived signals in early $\gamma$-MN development. We assessed the status of Wht7a in Pva::Cre; Isl2::DTA mutant mice, in which genetic elimination of proprioceptive sensory neurons leads to a failure in spindle differentiation (Hippenmeyer et al., 2002). In these mice, $98 \%$ of $\gamma$-MNs are lost by the second postnatal week (Friese et al., 2009), and accordingly we saw an absence of $W n t 7 a^{O N}$ motor neurons at P15 (Fig. 3C,F). We probed for Wnt7a expression in neonatal and early postnatal Pva::Cre; Isl2::DTA mutant mice and found a complete absence of Wnt7a expression at P0 (Fig. $3 A, D$ ) and P4 (Fig. $3 B, E)$. Because this may reflect abnormal $\gamma$-MN differentiation or alternatively, an absence of $\gamma$-MNs, we screened for expression of NeuN and ChAT. In P4 control mice, NeuN ${ }^{\mathrm{OFF}} / \mathrm{ChAT} T^{\mathrm{ON}} \gamma$-MNs intermingled with $\mathrm{NeuN}^{\mathrm{ON}} / \mathrm{ChAT}^{\mathrm{ON}}$ $\alpha$-MNs (79\%; $n=224$ of 285 ChAT $^{\text {ON }}$ motor neurons; Fig. 3J-J"). In contrast, Pva::Cre; Isl2::DTA mutant mice lacked NeuN ${ }^{\mathrm{OFF}} / \mathrm{ChAT}^{\mathrm{ON}} \gamma$-MNs while NeuN ${ }^{\mathrm{ON}} /$ ChAT ${ }^{O N} \alpha$-MNs $(96 \% ; n=181$ of 189 ChAT $^{\text {ON }}$ motor neurons; Fig. $\left.3 K-K^{\prime \prime}\right)$ persisted. These results suggest that a muscle spindle-derived signal is required for the development of $W n t 7 a^{O N} \gamma$-MNs in the first postnatal week. 


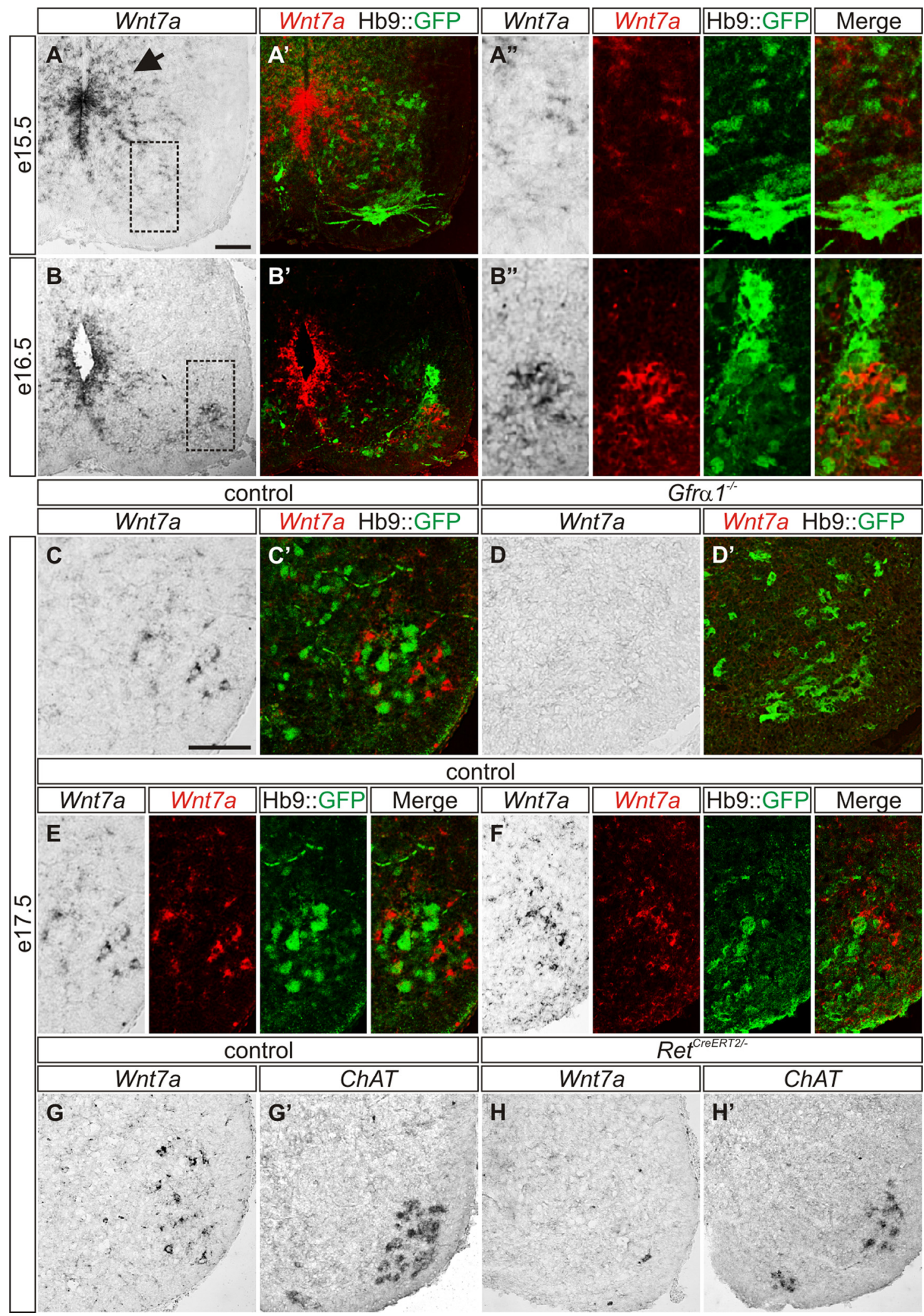

Figure 2. Wnt7A is expressed in embryonic $\gamma-M N s . A-A^{\prime \prime}$, Wnt7a is expressed in the ventricular zone (arrow) and is absent from the ventral motor neuron region in E15.5 Hb9::GFP mice $\left(\boldsymbol{A}, \boldsymbol{A}^{\prime}\right)$.

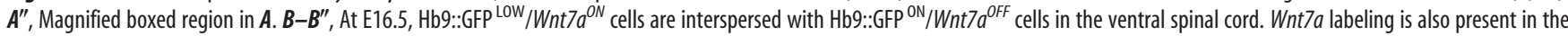
ventricular zone $\left(\boldsymbol{B}, \boldsymbol{B}^{\prime}\right) . \boldsymbol{B}^{\prime \prime}$, High magnification of boxed region in $\boldsymbol{B} . \boldsymbol{C}, \boldsymbol{C}^{\prime}, \boldsymbol{E}, \boldsymbol{F}, W n t 7 a^{\text {ON }}$ cells are localized throughout the motor neuron nucleus of E17.5 Hb9::GFP mice and are segregated from

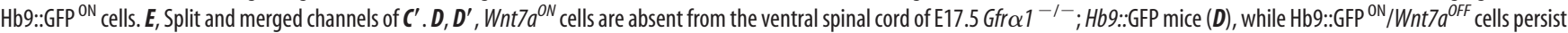
$\left(\boldsymbol{D}^{\prime}\right) . \mathbf{G}, \boldsymbol{H}^{\prime}, W n t 7 a^{\text {ON }}$ cells in the motor nucleus of E17.5 control mice $(\boldsymbol{G})$. Ret ${ }^{\text {(reeRT2/- }}$ mice lack Wnt7 $a^{\text {ON }}$ cells at E17.5 $(\boldsymbol{H})$. ChAT ${ }^{\text {ON }}$ cells in control $\left(\boldsymbol{G}^{\prime}\right)$ and Ret ${ }^{\text {(reeRT2/- }}$ mice $\left(\boldsymbol{H}^{\prime}\right)$. Split and merged channels shown for $\boldsymbol{A}^{\prime \prime}, \boldsymbol{B}^{\prime \prime}, \boldsymbol{E}$, and $\boldsymbol{F}$. Scale bars: $\boldsymbol{A}-\boldsymbol{H}^{\prime}, 100 \mu \mathrm{m}$. 

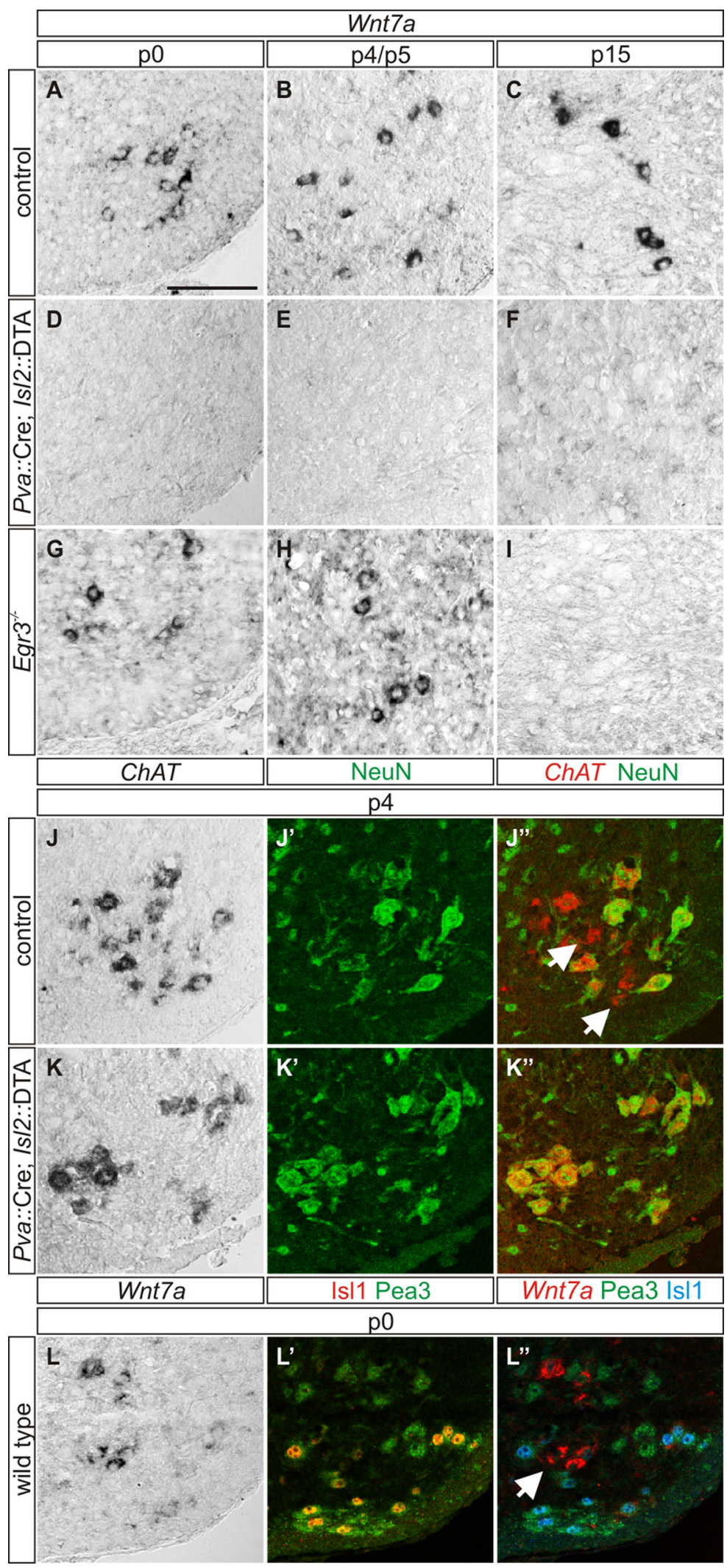

Figure 3. $A-C$, A spindle-derived GDNF-independent signal is required for $\gamma$-MN development. Wnt7 $a^{\text {ON }}$ putative $\gamma$-MNs are localized within the motor neuron nucleus of $\mathrm{PO}(\boldsymbol{A}), \mathrm{P} 4(\boldsymbol{B})$, and P15 ( $)$ control mice. $\boldsymbol{D}-\boldsymbol{F}$, Wnt7a labeling is eliminated in P0 (D), P4 (E), and P15 (F) Pva::Cre; Isl2::DTA mutant mice. G-I, Wnt7a ${ }^{\text {ON }} \gamma$-MNs are localized within the ventral spinal cord of PO (G) and P5 (H) Egr3 ${ }^{-/-}$mice, but

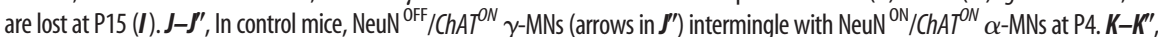

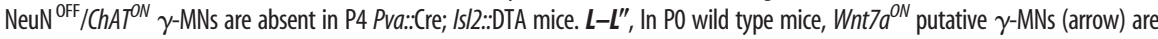
localized within the $\left(\mathrm{m}\right.$ motor pool, identified by coexpression of Islet1 and Pea3. Scale bar: $A-L^{\prime \prime}, 100 \mu \mathrm{m}$.
To assure that the absence of $W n t 7 a^{\text {ON }}$ putative $\gamma$-MNs in Pva::Cre; Isl2::DTA mutant mice is not due to the absence of central proprioceptive input, we analyzed the status of $\gamma$-MNs in the cutaneous maximus $(\mathrm{Cm})$ motor pool. Although these neurons normally do not receive proprioceptive input, Friese et al. (2009) showed that $\gamma$-MNs are present in this motor pool at P15. We identified Cm motor neurons at $\mathrm{P} 0$ using Islet $1{ }^{\mathrm{ON}} / \mathrm{Pea} 3{ }^{\mathrm{ON}}$ status (Vrieseling and Arber, 2006), and found that in wild type mice $W n t 7 a^{O N}$ $\gamma$-MNs are present in the Cm motor pool (Fig. $3 L-L^{\prime \prime}$ ). These findings suggest that the selective absence of $W n t 7 a^{O N} \gamma$-MNs in Pva::Cre; Isl2::DTA mice is not due to the loss of central proprioceptive input to motor neurons.

What is the identity of the signal controlling early $\gamma$-MN survival? Shneider et al. (2009) showed that in the second postnatal week, $\gamma$-MN survival depends on muscle spindle-derived GDNF. Egr3 is a transcription factor required for normal muscle spindle development and in its absence, muscle spindles degenerate during the first two postnatal weeks (Tourtellotte et al., 2001). GDNF fails to be expressed by muscle spindles of Egr3 ${ }^{-1-}$ mice (Shneider et al., 2009). We analyzed Egr3 ${ }^{-/-}$ spinal cords and found that there is a complete absence of Wnt7a signal in motor neurons by P15 (Fig. 3I), consistent with the previous demonstration that $\gamma$-MNs depend on muscle spindle derived GDNF for survival at this stage (Shneider et al., 2009). To test whether GDNF is similarly required for $\gamma$-MN survival shortly after birth, we analyzed $\mathrm{P} 0$ and $\mathrm{P} 5$ $\mathrm{Egr}^{-1-}$ mice and found that $\mathrm{Wnt} 7 \mathrm{a}^{\mathrm{ON}}$ cells are still present (Fig. 3G,H). Together these results demonstrate that independent of spindle-derived GDNF, a signal from the muscle spindle is required for the emergence of $W n t 7 a^{O N} \gamma$-MNs in the spinal cord.

\section{Discussion}

In the control of motor behavior, $\gamma$ - and $\alpha$-MNs have distinct functions that are reflected at the level of specific postnatal anatomical and molecular features. To further delineate the time course and mechanisms underlying $\gamma$ - and $\alpha-\mathrm{MN}$ diversification, we searched for markers that labeled these distinct motor neuron classes early during development. In this study, we found that Wnt7a is expressed in $\gamma$-MNs by E17.5, suggesting that these distinct motor neuron classes acquire different molecular identities before birth. We used Wnt7a expression as a marker to 
assess the role of target muscle spindles in $\gamma$-MN development and found that $\gamma$-MNs depend on a GDNF-independent muscle spindle-derived signal in the early postnatal period, revealing a novel target-derived trophic factor in $\gamma$-MN development.

The organization of motor neurons in the developing spinal cord is a highly regulated process. A cascade of intrinsic specification events and peripheral signals subdivides motor neurons into motor pools, each innervating a single muscle group (Lin et al., 1998; Jessell, 2000). One such peripheral signal, GDNF, has been shown to control the expression of the motor pool marker Pea3 both before and after motor neurons innervate their muscle targets (Haase et al., 2002; Livet et al., 2002). Evidence for a molecular distinction of $\gamma$ - and $\alpha$-MN classes has come from the identification of class-specific markers during the second postnatal week (Friese et al., 2009; Shneider et al., 2009). In the present study, we found that Wnt7a faithfully marks $\gamma$-MNs throughout postnatal development. Notably, we also found that Wnt7a specifically labels a subset of motor neurons at E17.5, well before the selective expression of Err3 and Gfr $\alpha 1$ in $\gamma$-MNs. In E17.5 Gfr 1 and Ret mutant mice, which lack $\gamma$-MNs, the Wnt7a signal was lost, confirming that Wnt7a is a marker of late embryonic $\gamma$-MNs.

What is the function of Wnt7a in $\gamma$-MNs? We analyzed Wnt7a mutant mice, but detected neither changes in the proportion and molecular identity of $\gamma$ - and $\alpha$-MNs nor in the synaptic input onto $\gamma$-MNs (S. Ashrafi and J. A. Kallschmidt, unpublished observation). We did however find that the size of both motor neuron classes was significantly reduced in Wnt7a mutants, which might be attributed to the wider distribution of Wnt7a expression during earlier stages of motor neuron development (Agalliu et al., 2009). We asked whether the expression of potential Wnt7a receptors might provide any clue toward the function of Wnt7a in $\gamma$-MNs and found that the Wnt7a receptor Frizzled 5 is expressed in muscle spindle capsules (S. Ashrafi and J. A. Kallschmidt, unpublished observation). However, we could not detect any defects in the innervation of intrafusal muscle spindles in Wnt7a mutant mice. Thus, although the identification of a secreted signaling molecule as an embryonic $\gamma$-MN marker is intriguing, the specific function of Wnt7a in $\gamma$-MNs remains unclear.

What are the mechanisms that drive the diversification of $\gamma$ and $\alpha$-MNs? Previous studies have shown that putative nascent $\gamma$-MNs are selectively dependent on GDNF signaling during embryonic development. In the absence of GDNF trophic support, nascent $\gamma$-MNs are lost during the normal period of programmed motor neuron cell death between E13.5 and E16.5 (Gould et al., 2008). Since the first molecular signs of muscle spindle differentiation are only observed by E15.5 (Hippenmeyer et al., 2002), this suggests that the initial specification of $\gamma$-MNs most likely occurs independently of the target spindle. During later postnatal development, GDNF produced by the target spindle is required for $\gamma$-MN survival (Shneider et al., 2009). However, because markers to identify $\gamma$-MNs during early postnatal development have not been available, it has been unclear whether muscle spindle-derived GDNF or other trophic signals may be required for early $\gamma$-MN differentiation. Here we found that $\gamma$-MN development in the first postnatal week depends on a muscle spindlederived signal. Shneider et al. (2009) showed that spindle-derived GDNF is not limiting in the first postnatal week suggesting that the early spindle dependence of $\gamma$-MNs is related to a targetderived factor other than GDNF. Consistent with this, we found that in P5 Egr3 mutant mice, in which muscle spindles are present but lack GDNF (Shneider et al., 2009), $\gamma$-MNs persist. Together these data suggest that a trophic signaling factor other than
GDNF may be the source for the signal for $\gamma$-MN differentiation during the early postnatal period. Support for this hypothesis is provided by a recent report demonstrating that distinct neurotrophic signaling pathways can operate to regulate the survival of subsets of motor neurons within a motor pool (Lamballe et al., 2011).

In summary, we propose a model in which multiple musclederived signals influence $\gamma$-MN development. Putative $\gamma$-MNs are first distinguished by a requirement for GDNF during the period of programmed cell death, when muscle spindles have not yet been induced by proprioceptor-derived signals (Gould et al., 2008). The mechanism that controls this initial specification of $\gamma$-MNs is not understood, but our finding of Wnt7a as an embryonic marker of fusimotor identity offers a means to explore this early motor neuron subclass distinction. $\gamma$-MNs then innervate nascent intrafusal muscle fibers and during early postnatal development require a non-GDNF, spindle-derived trophic signal. At 2 weeks postnatal, $\gamma$-MNs respond to GDNF, now supplied by the muscle spindle (Shneider et al., 2009), after which $\gamma$-MNs are independent of GDNF (Gould et al., 2008).

\section{References}

Agalliu D, Takada S, Agalliu I, McMahon AP, Jessell TM (2009) Motor neurons with axial muscle projections specified by Wnt4/5 signaling. Neuron 61:708-720.

Arber S, Han B, Mendelsohn M, Smith M, Jessell TM, Sockanathan S (1999) Requirement for the homeobox gene $\mathrm{Hb} 9$ in the consolidation of motor neuron identity. Neuron 23:659-674.

Burke RE, Strick PL, Kanda K, Kim CC, Walmsley B (1977) Anatomy of medial gastrocnemius and soleus motor nuclei in cat spinal cord. J Neurophysiol 40:667-680.

Chakkalakal JV, Nishimune H, Ruas JL, Spiegelman BM, Sanes JR (2010) Retrograde influence of muscle fibers on their innervation revealed by a novel marker for slow motoneurons. Development 137:3489-3499.

Enjin A, Rabe N, Nakanishi ST, Vallstedt A, Gezelius H, Memic F, Lind M, Hjalt T, Tourtellotte WG, Bruder C, Eichele G, Whelan PJ, Kullander K (2010) Identification of novel spinal cholinergic genetic subtypes disclose Chodl and Pitx2 as markers for fast motor neurons and partition cells. J Comp Neurol 518:2284-2304.

Enomoto H, Araki T, Jackman A, Heuckeroth RO, Snider WD, Johnson EM Jr, Milbrandt J (1998) GFR alphal-deficient mice have deficits in the enteric nervous system and kidneys. Neuron 21:317-324.

Enomoto H, Hughes I, Golden J, Baloh RH, Yonemura S, Heuckeroth RO, Johnson EM Jr, Milbrandt J (2004) GFRalphal expression in cells lacking RET is dispensable for organogenesis and nerve regeneration. Neuron 44:623-636.

Friese A, Kaltschmidt JA, Ladle DR, Sigrist M, Jessell TM, Arber S (2009) Gamma and alpha motor neurons distinguished by expression of transcription factor Err3. Proc Natl Acad Sci U S A 106:13588-13593.

Gould TW, Yonemura S, Oppenheim RW, Ohmori S, Enomoto H (2008) The neurotrophic effects of glial cell line-derived neurotrophic factor on spinal motoneurons are restricted to fusimotor subtypes. J Neurosci 28:2131-2146.

Haase G, Dessaud E, Garcès A, de Bovis B, Birling M, Filippi P, Schmalbruch $\mathrm{H}$, Arber S, deLapeyrière O (2002) GDNF acts through PEA3 to regulate cell body positioning and muscle innervation of specific motor neuron pools. Neuron 35:893-905.

Hippenmeyer S, Shneider NA, Birchmeier C, Burden SJ, Jessell TM, Arber S (2002) A role for neuregulin 1 signaling in muscle spindle differentiation. Neuron 36:1035-1049.

Hippenmeyer S, Vrieseling E, Sigrist M, Portmann T, Laengle C, Ladle DR, Arber S (2005) A developmental switch in the response of DRG neurons to ETS transcription factor signaling. PLoS Biol 3:e159.

Jessell TM (2000) Neuronal specification in the spinal cord: inductive signals and transcriptional codes. Nat Rev Genet 1:20-29.

Kanning KC, Kaplan A, Henderson CE (2010) Motor neuron diversity in development and disease. Annu Rev Neurosci 33:409-440.

Kuffler SW, Hunt CC, Quilliam JP (1951) Function of medullated smallnerve fibers in mammalian ventral roots; efferent muscle spindle innervation. J Neurophysiol 14:29-54. 
Lamballe F, Genestine M, Caruso N, Arce V, Richelme S, Helmbacher F, Maina F (2011) Pool-specific regulation of motor neuron survival by neurotrophic support. J Neurosci 31:11144-11158.

Lin JH, Saito T, Anderson DJ, Lance-Jones C, Jessell TM, Arber S (1998) Functionally related motor neuron pool and muscle sensory afferent subtypes defined by coordinate ETS gene expression. Cell 95:393-407.

Livet J, Sigrist M, Stroebel S, De Paola V, Price SR, Henderson CE, Jessell TM, Arber S (2002) ETS gene Pea3 controls the central position and terminal arborization of specific motor neuron pools. Neuron 35:877-892.

Luo W, Enomoto H, Rice FL, Milbrandt J, Ginty DD (2009) Molecular identification of rapidly adapting mechanoreceptors and their developmental dependence on ret signaling. Neuron 64:841-856.

Parr BA, McMahon AP (1995) Dorsalizing signal Wnt-7a required for normal polarity of D-V and A-P axes of mouse limb. Nature 374:350-353.

Peljto M, Dasen JS, Mazzoni EO, Jessell TM, Wichterle H (2010) Functional diversity of ESC-derived motor neuron subtypes revealed through intraspinal transplantation. Cell Stem Cell 7:355-366.

Romanes GJ (1951) The motor cell columns of the lumbo-sacral spinal cord of the cat. J Comp Neurol 94:313-363.

Sabharwal P, Lee C, Park S, Rao M, Sockanathan S (2011) GDE2 regulates subtype-specific motor neuron generation through inhibition of Notch signaling. Neuron 71:1058-1070.

Schuchardt A, D’Agati V, Larsson-Blomberg L, Costantini F, Pachnis V (1994) Defects in the kidney and enteric nervous system of mice lacking the tyrosine kinase receptor Ret. Nature 367:380-383.

Shneider NA, Brown MN, Smith CA, Pickel J, Alvarez FJ (2009) Gamma motor neurons express distinct genetic markers at birth and require muscle spindle-derived GDNF for postnatal survival. Neural Dev 4:42.

Tourtellotte WG, Milbrandt J (1998) Sensory ataxia and muscle spindle agenesis in mice lacking the transcription factor Egr3. Nat Genet 20:87-91.

Tourtellotte WG, Keller-Peck C, Milbrandt J, Kucera J (2001) The transcription factor Egr3 modulates sensory axon-myotube interactions during muscle spindle morphogenesis. Dev Biol 232:388-399.

Vrieseling E, Arber S (2006) Target-induced transcriptional control of dendritic patterning and connectivity in motor neurons by the ETS gene Pea3. Cell 127:1439-1452.

Wichterle H, Lieberam I, Porter JA, Jessell TM (2002) Directed differentiation of embryonic stem cells into motor neurons. Cell 110:385-397.

Yang X, Arber S, William C, Li L, Tanabe Y, Jessell TM, Birchmeier C, Burden SJ (2001) Patterning of muscle acetylcholine receptor gene expression in the absence of motor innervation. Neuron 30:399-410. 